\title{
Editoríal.
}

\section{THE NEUROSES.}

$\mathrm{N}^{2}$

OTWITHSTANDING advances that are taking place all along the front of medicine, it is a question whether present-day conceptions of what àre currently denominated 'neuroses' and 'psychoneuroses' are as serviceable as is often claimed. To classify and differentiate the neuroses has ever been troublesome, mainly though not entirely owing to etiological uncertainty. Old distinctions between 'functional' and 'organic' types of symptom have proved valueless except for clinical diagnosis, and in this field also their usefulness is over. If the Babinski variety of plantar reflex response characterises a series of conditions whose basis is an actual structural change in cerebrospinal tracts, it is also found, unmistakably, under circumstances which no one can say represent other than transient disorders of neural function. For a few minutes after a major epileptic attack the plantar response may assume the form of typical great-toe extension. From the standpoint of causation, therefore, a test so well-recognised and constantly employed as that of plantar stimulation cannot render any aid.

It may become difficult, accordingly, to find objective criteria whereby to define and determine a ' neurosis.' Theoretically and etymologically, the term, as Dr. Millais Culpin urges, should indicate a disturbance of the intrinsic function of nerve tissuc - it is pointless otherwise. But what is this intrinsic function, we may well enquire ? Excitation and inhibition are the physiological phenomena of neural tissue; stimuli applied to the nervous system elicit responses which assume the form of the one or the other, or of their succession, nor can any other variety be obtained. Thus considered, any nourological syndrome is a neurosis. The vicissitudes through which the word has passed, however, impress us to-day with the desirability of a certain restriction ; we find, in point of fact, that it is commonly utilised to signify disorders of function for which, by way of cause, no recognisable physiological disability can be detected. In the seeming absence of such etiology, present-day doctrine 
declares for a psychological origin, and some, e.g., Dr. Culpin, go so far as to consider neurosis synonymous with ' minor psychosis.' Others advance further; Dr. Israel Wechsler includes the psychoses (paraphrenia, schizophrenia, manicdepressive psychosis, and paranoia) under the term ' narcissistic neuroses.'

Here, surely, is confusion confounded. And yet the steps whereby so unsatisfying a nomenclature is reached can be readily followed. For the truth is that 'neurosis' is merely a name, an attempt to describe (for the sake of the clinician) a series of states which it is thought must be called something and might as well have that label attached as any other. In the consideration of clinical conditions for which the designation seems appropriate two matters have to be examined-firstly, the causation, and secondly, the intrinsic nature of the symptoms. Now as regards the former of these, it behoves the critical mind to proceed warily. The terminologists of the day appear with some unanimity to accept say writers' cramp as an 'occupation neurosis' and to argue for its inclusion because ' examination and experience tell us that its subject has no physiological disability of nerve.' It is alleged to be analogous to stammering -and that is of psychogenic derivation, we are assured. Butक so far as the manifestations of writers' cramp are concerned, they are in fact capable of being described as expressive of either increased excitability and irritability, on the one hand, or of augmented inhibition on the other. That is to say, thein intrinsic features indicate disorder of physiological function,? postulated psychical origin notwithstanding. Thus a case might be made out for the view that this particular ' neurosis' may develop on a basis of inherent defect of neural function, showing itself only when a given set of circumstances (e.g., long repetition of the same muscular act) is in being. Or take ' la grande névrose' of our French colleagues, the hysteria whose fundamental problems, pace the psychoanalysts, have not yet been solved. If a patient cannot move his arm, which hangs flaccid from the shoulder, and if examination discloses no change in electrical reactions or reflectivity, are we to say that its physiological function is normal and that the condition constitutes a neurosis, a 'minor psychosis'? On the contrary, from the viewpoint of physiology it is one of pure inhibition. The person concerned cannot innervate his brachial neuromuscular units ; there is a block somewhere. What boots it to ascribe dogmatically the impasse to the psyche? This is surely an assumption of an unwarranted kind. For we do not 
know what the psyche stands for in physiological terms, and if the psychologist smiles at the naivété of imagining the two systems comparable it is none the less conceivable (evidence pointing in this direction might be adduced) that the ' will' is nothing else than de-inhibition, and that the failure of the hysteric to innervate his arm is a question of physiological incapacity to remove inhibitory impediment. That this neural defect-for such it is - should be conditioned by a disorder of the psychical series may be the case ; the symptom arises, at any rate, on a mechanistic basis capable of physiological description. Nor must we too rashly conclude that were a psychical trauma or other such impairment to be discovered in the patient's mental history the exteriorisation of the syndrome is thereby explained.

No less deserving of reconsideration is the term 'psychoneurosis,' to find a proper justification for which would be a matter of no little arduousness. Like 'psychomotor,' 'psychosensory,' 'psychovisual,' and others, it represents a blend of physiological and psychological without any definition of the assumed interaction or interrelation. If it stands for something a little more than neurosis, a little less than psychosis, its descriptive value is outweighed by its nosological impropriety. For, as we have seen, one of the disciples of present-day modernism (the collocation is intentional and meaning-full) takes neurosis to signify psychosis - ' minor,' if you will, but who is to define 'minority'?

Until some agreement is attained in respect of the principles on which our terminology in medicine is to be established confusion in the names given to things will not be dispelled; further, persistence in employment of inappropriate designations will continue to imply the existence of unfounded etiological conceptions. The word 'neurosis' implies disorder of neural function-it is therefore essentially unspecific ; if it entails the admission of a psychical causation it is unsuitable ; and so long as it is imagined to stand for physiological disorder with physiological integrity it is meaningless. Of more immediate practical importance than all such discussions is the problem whether any particular physiological characters distinguish the neurosis from other functional imperfections. This aspect of the matter, still largely unnoticed, demands painstaking research. Even those who contend stoutly for the psychological origin of the neuroses (as they understand the term) must naturally admit the frequency with which actual interference with physiological mechanisms occurs. The 'anxiety neurosis' of current textbooks is compact of recognisable and definable somatic and visceral disturbances. 
In themselves, have these such a stamp, do they run such a course, or exhibit such intrinsic features, as to enable the physician to separate them from other involvements of the same mechanisms ? Can any peculiarities be discovered in its physiological manifestations to differentiate hysteria from other states ? The limited possibilities of the psychical-sexual origin of hysteria must contrast strikingly, in the mind of any unbiased observer, with the remarkable variability of its manifold clinical phenomena. Were any specificity to attach to these, from a neural point of view, the inadequateness of psychogunic theory would be patent. The more decply clinical observation penetrates the arcana of the neuroses the more possible does it seem that the class as a whole will suffer dismemberment. Chorea was once a neurosis, and paralysis agitans! Have we finished with telegraphists' cramp when we note its occurrence in a 'neurotic' person, with ' nervous heart,' bad dreams, fatigability, fears and what not? What if these are themselves but the expression of physiological disarray? 